\title{
Investigation of the Interaction between Coumarins and Its Derivatives with Human Serum Albumin: STD-NMR, Fluorescence and Docking Molecular Studies
}

\author{
Fernanda Paulin Benzatti ${ }^{1}$, Ícaro P. Caruso1, Thiago Salem \\ Pançonato Teixeira ${ }^{2}$, Fábio Rogério Moraes ${ }^{1}$, Fernando Alves de \\ Melo1, Luiz Claudio Di Stasi³, Marinônio Lopes Cornélio', Fatima \\ Pereira de Souza4, Marcelo Andres Fossey ${ }^{1}$
}

\author{
${ }^{1}$ CMIB/Physics Departament, IBILCE, UNESP \\ ${ }^{2}$ CMIB-IBILCE, UNESP \\ ${ }^{3}$ Pharmacology Departament, IBB, UNESP \\ ${ }^{4}$ Fátima Pereira de Souza, Instituto de Biociências, Letras e CiênciasExatas - IBILCE/UNESP. RuaCristóvão \\ Colombo, 2265. São José do Rio Preto, SP, Brazil. CEP15054-000
}

\begin{abstract}
In this paper, binding interaction of Coumarin, including 4-Methylesculetin, Esculetin and Esculin, with human serum albumin (HSA) was investigated by using STD-NMR, fluorescence spectroscopy and molecular docking method. STD-NMR investigations indicated that the binding affinity sequence for HSA-ligands interaction was: 4-Methyllesculetin >Esculetin>Coumarin>Esculinbeingin accordance with the fluorescence studies. The molecular docking resultssuggested that coumarins and its derivatives were binding to HSA at subdomain IIA, nearby the Trp214 residue, which are consistent with the results of fluorescence quenching results. Overall, the experimental and theoretical data corroborate with each other and they are complementary.
\end{abstract}

Keywords: Coumarins and their derivatives, Human Serum Albumin, STD-NMR, fluorescence quenching, docking molecular.

\begin{abstract}
Introduction
Coumarins comprise a very large class of compounds that occur naturally in plants [1]. Thesecompounds are lactones of o-hydroxy-cinnamic acid with typical benzopyrone framework. Coumarin and their derivatives have roles as anti-inflammatory, anticoagulant, anti-retroviral, anti-arthritic, anti-asthmatic $[2,3]$, antioxidant and intestinal anti-inflammatory activities [4,5]they can also cause significant changes in the regulation of the immune response, cell growth, and differentiation [6]. The mode and strength of coumarin activity usually could be modified by the number and type of small substituent, namely hydroxy or methoxy groups [1]. Coumarins have recently attracted much attention due to their broad pharmacological activities as precursor molecules for the synthesis of number of synthetic anticoagulant
\end{abstract}

drugs [7]. The structural studies of complexes formed by simple coumarins with diverse proteins indicated that the nature of ligand-protein binding is a combination of hydrophobic, electrostatic and/or hydrogen bonding interactions [8]. Several coumarins derivatives are bound to serum proteins, especially human serum albumin (HSA). HSA, the most abundant and the major transport protein in the blood, is used as a carrier in the transport of various endogenous and exogenous compounds throughout the body until their target organs[9]. It greatly influences drug distribution and can play a major role in affecting drug absorption, distribution, metabolism, and excretion [10]. It is a globular protein synthesized and secreted from liver cells and contains 585 aminoacid residues with a total molar mass of $66 \mathrm{kDa}$. It has three homologous helical domains (I-III), each divided into $\mathrm{A}$ and $\mathrm{B}$

This article is published under the terms of the Creative Commons Attribution License 4.0

Author(s) retain the copyright of this article. Publication rights with Alkhaer Publications.

Published at: http://www.ijsciences.com/pub/issue/2016-02/

DOI: 10.18483/ijSci.777; Online ISSN: 2305-3925; Print ISSN: 2410-4477 
Investigation of the Interaction between Coumarins and Its Derivatives with Human Serum Albumin: STD-NMR, Fluorescence and Docking Molecular Studies

subdomains. Aromatic and heterocyclic ligands have been found to bind mainly within subdomains IIA and IIIA designated as site I and site II, respectively. It has only one tryptophan at position 214 in subdomain IIA [11] that possess intrinsic fluorescence,thus, fluorescence measurement is used as a probe for the drug binding studies with HSA [12].

Understand the interaction between drugs and the plasma proteins is essential because this binding affects their pharmacodynamic and pharmacokinetic properties and can make us understand the absorption and distribution of drug [13].A wide variety of biophysical methods have been used for the analysis of the interactions between protein and ligands, such as, fluorescence spectroscopy[14], fluorescence resonance energy transfer (FRET)[15,16],nuclear magnetic resonance (NMR) [17], isothermal titration calorimetry (ITC)[18], besides computational methods/ molecular modeling [19].

Currently, some researchers have been used the results obtained from STD-NMR and fluorescence studies to complement the molecular modeling of the coumarins and getting a much clearer picture of the binding process in HSA [20], thus in this paper we will discuss only the interactions observed through the techniques STD-NMR, fluorescence spectroscopy and molecular docking method.

The purpose of the present study is to investigate the binding ability of the ligands Coumarin (1,2benzopyrone) and three substituted derivatives of it, 4Methylesculetin (6,7-dihydroxy-4-methylcoumarin), Esculetin (6,7-dihydroxy-coumarin) andEsculin with HSA using STD-NMR, fluorescence spectroscopy and molecular docking method.

\section{Materials and Methods}

Materials and Sample preparation

Coumarins and their derivatives, 4-Methylesculetin, Esculin and Esculetinwere purchased from SigmaAldrich. The protein, HSA fraction V, was also purchased from Sigma-Aldrich. For UV measurements and Fluorescence spectroscopy, HSA solution was freshly prepared before the experiments in $50 \mathrm{mM}$ phosphate buffer ( $\mathrm{pH} 7.40)$ at $298 \mathrm{~K}$. Stock solutions of Coumarin (MM 146,14 Da) and its derivatives, 4Methylesculetin (MM 192,17 Da), Esculetin (MM $178,14 \mathrm{Da})$ and Esculin (MM 340,28 Da) were prepared in $95 \%$ ethanol. For NMR studies,it was prepared a $5 \mu \mathrm{M}$ HSA solution in deuterium oxide and $50 \mathrm{mM}$ mono-sodium phosphate buffer, pD of 7.4. Each ligand was solubilized in deuterated ethanol at the following stockconcentrations: 4-Methylesculetin $45 \mathrm{mM}$, Esculetin $40.5 \mathrm{mM}$, Coumarin $24 \mathrm{mM}$ andEsculin $40 \mathrm{mM}$. Both protein and ligands were stored at $4{ }^{\circ} \mathrm{C}$ before use.
UV spectra measurements

The UV absorbance spectra were recorded using a CARY-3E UV-Vis spectrometer (Varian, USA) equipped with $1.0 \mathrm{~cm}$ quartz cells at $298 \mathrm{~K}$.

HSA concentration was estimated from its UV absorption using an extinction coefficient $(\varepsilon)$ of 36,850 $\mathrm{M}^{-1} \mathrm{~cm}^{-1}$ at $280 \mathrm{~nm}$ [25]. The stock solution concentration of Coumarin was calculated using molar extinction coefficient $\varepsilon_{277}=11.438 \mathrm{M}^{-1} \mathrm{~cm}^{-1}$ at $277 \mathrm{~nm}$, 4-Methylesculetin $\varepsilon_{340}=11.062 \mathrm{~cm}^{-1} \mathrm{M}^{-1}$ at375 $\mathrm{nm}$, Esculetin $\varepsilon_{344}=10.191,1 \quad \mathrm{~cm}^{-1} \quad \mathrm{M}^{-1}$ at $344 \mathrm{~nm}$ and Esculin $\varepsilon_{344}=11.466 \mathrm{~cm}^{-1} \mathrm{M}^{-1}$ at $344 \mathrm{~nm}$, all of them were calculated by $\mathrm{UV}-\mathrm{V}$ is spectrophotometric.

\section{STD-NMRspectroscopy}

NMR spectra were recorded on a BrukerAvance III $600.13 \mathrm{MHz}$ equipped with a triple resonance $5 \mathrm{~mm}$ cryo-probe, having pulse filed gradient along the $\mathrm{Z}$ axis. All data were analyzed with Bruker Topspin 3.2 version. The HSA solution was used for determining the best saturation condition. It was tested with different frequencies at $-0.5,-1.0$ and $-1.5 \mathrm{ppm}$, keeping the off-resonance frequency at $40 \mathrm{ppm}$. Protein saturation time was established at $2 \mathrm{~s}$, recycle delay at 2 s, a total of 1024 scans were collected with 4 dummy scans and the saturation power was set to -35 $\mathrm{dBW}$. When adding each ligand individually a spin lock filter of $30 \mathrm{~ms}$ was used for protein signals suppression. Competition experiments are known to result in direct affinity comparison among two ligands [26]. In that way, it was performed competition experiments among pairs of coumarins, in order to get insight into the affinity comparison.

\section{Fluorescence spectroscopy}

The fluorescence measurements were performed using an ISS PC1 steady-state spectrofluorimeter (Champaign, IL, USA) equipped with a Neslab RTE221 thermostat bath. Both excitation and emission bandwidths were set at $4.0 \mathrm{~nm}$. The excitation wavelength at $295 \mathrm{~nm}$ was chosen since it provides no excitation of tyrosine residues, but excites the single tryptophan residue (Trp214) of HSA. The emission spectrum was collected in the range of $305-500 \mathrm{~nm}$ which was corrected for the background fluorescence of the buffer and for inner filter effects [27]. In the fluorescence quenching experiments performed at 298 $\mathrm{K}$, the titrations were done by adding small aliquots (of $2.5 \mu \mathrm{M}$ ) from ligand stock solutions to HSA solution $(3 \mathrm{~mL}$ )at constant concentrations of $10 \mu \mathrm{M}$. The final concentration of each ligand achieved with the titrations was $25 \mu \mathrm{M}$.In all experiments, the final ethanol concentration in buffer was $<1 \%$. 
Investigation of the Interaction between Coumarins and Its Derivatives with Human Serum Albumin: STD-NMR, Fluorescence and Docking Molecular Studies

Molecular Modeling and Docking

Preparation of receptor molecules: 3D crystal structure of HSAwas downloaded from the Protein Data Bank (PDB) [28] structural database site (PDB code: 1AO6)[29]. Ligand preparation: The 2D structure of 4Methylesculetin (CID 5319502), Esculetin (CID 5281416), Coumarin (CID 323) and Esculin (CID 5281417) were retrieved from the PubChem database.

Docking methodology: Auto Dock Tools 4.0 [30] was used to prepare, run and analyze the docking simulations. The grid map was built centralized on Trp214 with grid box size of $50 \times 50 \times 50$ points and $0.375 \AA$ spacing. The Lamarckian Genetic Algorithm search parameter was used with number of GA runs of 100 , population size of 300 and maximum number of evaluations medium. The remaining was kept as default. All the auto dock docking runs were performed in Intel Core 2Duo, 64 bit operating system and 4GB RAM in Dell Vostro 1520.

\section{Results and discussions}

STD-NMR spectroscopy

Nuclear Magnetic Resonance (NMR) methods are used to study molecular interactions and it became essential in the investigation of ligand-protein interactions [21,22] (Meyer and Peters, 2003) (Lepre et al 2004). Saturation Transfer Difference (STD) [23] (Mayer and Meyer, 1999) experiments are increasingly being used for such purpose. The key idea behind STD-NMR spectroscopy is that Nuclear Overhauser Effect (NOE) signals from large molecules are negative while from small molecules (up to $\sim 1.2 \mathrm{kDa}$ ) are positive. If interaction takes place, the ligand receives the magnetization from the protein and leaves the binding pocket carrying its negative NOE. Following, the intensity of the ligands signals may be observed when subtracting the onresonance spectrum from the reference spectrum, since the negative NOE reduce the intensity of the ligand signals [23]

Figure 1-a and Figure 1-b present four superposed different spectra of the STD experiments with each ligand. It informs that 4-Methylesculetin, Esculetin and Coumarin specifically interact with HSA, while Esculin shows rather low intensity signals. The intensity of each hydrogen atom is presented as color coded in figure 1-b, and numbering may be associated with figure 1-c for each ligand. The ligand binding epitopes may also be evaluated from single STD experiment [24]. This is related to how close the small molecule approaches its receptor, and it is achieved by comparison of the relative transfer to different protons from the ligand in the difference spectrum. Several ligand types as peptides, carbohydrates have been shown good STD-NMR responses, allowing to correctly assess not only the binding event also the geometry of the protein-ligand complex under study (Mayer and Meyer, 2001). Within the STD-NMR framework, the binding epitopes for the ligands are evaluated by comparing each ligand atom intensity from the reference spectra to the difference spectra. What is achieved is that the higher the ratio between the intensity in the difference spectrum as compared to the intensity in the reference spectrum the more buried the region of the ligand is in the binding pocket. It arises from the fact that intermolecular NOE depends on the inverse sixth power of the nuclei distances. Furthermore, the ligand protons closer to the protein protons will have higher relative intensities in the difference spectrum [31].

The binding epitopes of the ligands in complex HSA are shown in Figure 1-c that informs the relative percentage of each chemical group. The integral value of the largest signal in the difference spectrum is associated to $100 \%$ transfer and more modest contributions are given as ratios from this intensity. Remarkably, all ligands show the same binding epitope pattern, and the predicted binding mode is presented. The ligand region between carbons C3 and C8 are understood as the driving component of each ligand until the HSA interaction site, as they are readily observed as relative intensity of the largest $(100 \%)$ and second largest within all ligands. Then, STD-NMR spectroscopy is able to distinguish between regions of the ligands in close contact to the protein receptor from the other hydrogen atoms present in the molecule. This result is used to filter out improper molecular docking poses, in order to achieve better visualization of the proposed binding mode.Also, competition experiments reveal the ranking affinity among the coumarins binding to HSA. When using 4-Methylesculetin and Esculetin, it was observed a slightly favored binding towards the former. When competing Esculetin against Coumarin, it is perceived that Esculetin has higher saturation transferred, which in principle gives higher affinity. Nevertheless, Esculin presents such a weak binding and no competition was performed. The affinity rank of the ligands under study binding with HSA is: 4-Methylesculetin $>$ Esculetin $>$ Coumarin $>$ Esculin.

\section{Fluorescence quenching process of HSA by ligands}

Fluorescence quenching refers to any process which decreases the fluorescence intensity of a sample. A diversity of molecular interactions may result in quenching, among them reactions with excited state, molecular rearrangement, energy transfer, ground state complex formation and collisional quenching. The different mechanisms of quenching are usually classified as either dynamic or static[32]. Figure2 shows thatthefluorescence intensity of HSA decreases with increasing concentration of various ligands, 
indicating that their microenvironment of the interaction are around the single tryptophan residue (Trp214) within the hydrophobic pocket of subdomain IIA, site 1 of HSA, thus quenching the fluorescence intensity emitted by Trp214 residue.

The data analyses regarding fluorescence quenching process was performed applying SternVolmer equation [32]:

$$
F_{0} / F=1+K_{q} \tau_{0}[Q]=1+K_{S V}[Q](1)
$$

where $F_{0}$ and $F$ are the steady-state fluorescence intensities in the absence and presence of quencher ( $Q)$, respectively. $K_{q}$ is the bimolecular quenching constant, $\tau_{0}$ is the fluorophore lifetime in the absence of quencher which value for HSA is $10^{-8} \mathrm{~s}[33],[Q]$ is the quencher concentration and $K_{S V}$ is the Stern-Volmer quenching constant.Figure 3 shows that within the investigated concentration range, the results of fluorescence quenching at $298 \mathrm{~K}$ are in accordance with the Stern-Volmerequation.

The results in Table 1 show that the Stern-Volmer constant values $\left(K_{S V}\right)$ present the following order of magnitude: 4-Methylesculetin $>$ Esculetin>Coumarin>Esculin.This result is in agreement with the one observed in the STDNMR, confirming that both techniques are effective in determining the molecules binding affinity.The bimolecular quenching constants $\left(K_{q}\right.$ ) calculated by $K_{q}=K_{S V} / \tau_{0}$ also are shown at Table 1. The $K_{q}$ values for 4-Methylesculetin, Esculetin and Coumarin are greater than $2.0 \times 10^{10}$ $\mathrm{M}^{-1} \cdot \mathrm{s}^{-1}$, whichrepresent the maximum diffusion collision rate of various quenchers with biopolymers[38], while for Esculin, $K_{q}$ has the same order of magnitude.Thus, the fluorescence quenching mechanism of HSA by 4Methylesculetin, Esculetin and Coumarin,should follow the static quenching process, whereas for Esculin, the dynamic process. Consequently, the formation of HSA-ligand complex should occurs onlyfor 4-Methylesculetin, Esculetin and Coumarin.

\section{Distance Measurement between HSA-Trp214 and Ligands}

Fluorescence resonance energy transfer (FRET) is a spectroscopic method that can monitor the proximity and relative angular orientation of fluorophores. According to Förster non- radioactiveenergy transfer theory, the energy transfer will occur under the following conditions: the donor emits fluorescent light; the emission spectrum of the donor and the absorbance spectrum of the acceptor have a partial overlap; orientation of the transition dipole of the donor and acceptor; and the distance between the donor and acceptor is less than $8.0 \mathrm{~nm}$ [32]. According to this theory, the average distance $r$ in the binding between HSA-Trp214 (donor) and ligands (acceptor) can be calculated by the equation:

$$
E=1-F / F_{0}=R_{0}^{6} /\left(R_{0}^{6}+r^{6}\right)
$$

where $E$ is the efficiency of energy transfer and $R_{0}$ is the critical distance when the transfer efficiency is $50 \%$.

$R_{0}^{6}=8.79 \times 10^{-25} K^{2} n^{-4} \phi J$

In Equation (3), $K^{2}$ is the orientation space factor, $n$ is the refracted index of the medium, $\phi$ is the fluorescence quantum yield of the donor, $J$ is the effect

$t$ of the spectral overlap between the emission spectrum of the donor and the absorption spectrum of the acceptor (Fig. 4), which may be calculated by the following equation:

$J=\int_{0}^{\infty} F(\lambda) \varepsilon(\lambda) \lambda^{4} d \lambda / \int_{0}^{\infty} F(\lambda) d \lambda$

where $F(\lambda)$ is the corrected fluorescence intensity of the donor in the wavelength range from $\lambda$ to $\lambda+\Delta \lambda$, and $\varepsilon(\lambda)$ is the extinction coefficient of the acceptor at $\lambda$.

In this study, $K^{2}=2 / 3, n=1.36$, and $\phi=0.074$ [34]. According to Equations (2) - (4), it was calculated the values of $J, E, R_{0}$ and $r$ (Table $2)$. The average distances between donor and acceptor fluorophore is in the range $0.5 R_{0}<r<1.5 R_{0}$, except to Esculin. The donor-acceptor distances found in this range (4Methylesculetin, Esculetin and Coumarin) indicate that the energy transfer from HSA to ligands likely may occur.

Docking Molecular 
The molecular docking calculations generated 100 poses for each ligand. From usual cluster analysis obtained for the molecular docking results, it were selected the conformers with the lowest binding energy within the largest cluster generated. The theoretical binding energy sequence obtained for these conformers (4-Methylesculetin $>$ Coumarin $>$ Esculin $>$ Esculetin) is not in agreement with the experimental results (Supplementary material, Table S1). Based on this usual analysis, the arrangement observed for the ligands in the binding site of HSA (Supplementary material, Fig. S1 and Fig. S2) does not correspond to the binding mode proposed by STD-NMR binding epitopes data (section 3.1).

Because of the disagreement between theoretical and experimental binding energy sequence, it was performed an evaluation of the contact distances between ligands and binding site of HSA. The contact distance criterion $(\leq 5 \AA)$ was used in order to follow the distance dependence $\left(\sim 1 / \mathrm{r}^{6}\right)$ that NOE presents in the STD experiments [17]. Since STD-NMR binding epitopes evaluation revealed the hydrogen atoms from $\mathrm{C} 3$ and $\mathrm{C} 8$ as the most buried groups in the protein binding site (Fig. 1), the average distance measurements for each ligand pose were performed from the $\mathrm{O} 2$ oxygen atom (middle point between both epitopes) to binding site atoms. The conformers with the lowest average contact distances that followed experimental affinity sequence were selected to evaluate the interactions involved in the binding site of HSA (Fig. 5).

4-Methylesculetin, Esculetin and Coumarin accommodates in a pocket made by the amino acids Lys195, Leu198, Lys199, Ser202, Ala210, Phe211, Trp214, Val344, Leu347, Asp454, Ser454, Leu481 and Val482. The main forces that stabilize these ligands is hydrophobic interaction, although for 4-Methylesculetin and Esculetin is observed a hydrogen interaction. In a minor extension, electrostatic interaction also contributes to the interaction.

On the other hand, esculin docked in a pocket on HSA surface, made by amino acids Arg209, Lys212, Ala213, Asp324, Leu327, Gly328, Leu347, Ala350, Lys351, Glu354 and Val482. This molecule interacts with the protein mainly by hydrophobic interaction and in a minor extension by hydrogen interaction and electrostatic interaction.

\section{Conclusion}

In this study, the experimental techniques of STDNMR and fluorescence spectroscopy along with molecular docking calculations were used to investigate the interaction of the HSA with Coumarin and its derivatives.STD-NMR experiments were used for group epitope mapping [24], i.e., the identification of the ligand most buriedprotons in the protein binding site.The STDNMR experiments demonstrated the following binding affinity sequence for HSA-ligands interaction: 4-Methyllesculetin $>$ Esculetin $>$ Coumarin $>$ Esculin. The same result was observed in the fluorescence quenching experiments, which indicate that the ligands are located close to the Trp214 residue in subdomain IIA of HSA.Substituents on the ring of Coumarin molecule (hydrophobic portion) (Fig. 1)play an important role on the activity of the molecule[5]. The binding affinity is not directly related to hydrophobicity or hydrophobicity of substituents on the ring, however, it has an indirect role by affecting the intramolecular interaction in the molecule [35].The coumarin derivatives have the same origin functional group (benzopyrone), but with different substituents at different positions. The presence of a methyl group at $4^{\text {th }}$ position and two hydroxyl groups at $6^{\text {th }}$ and $7^{\text {th }}$ position of the Coumarin molecule (4-Methyllesculetin) resulted in a higherbinding affinity of this compound with HSAthan only the substitution of hydroxyl groups (Esculetin). However, the presence of a glucose and hydroxyl group at $6^{\text {th }}$ and $7^{\text {th }}$ position (Esculin), respectively, results in a very weak interaction of this coumarin derivative with the HSA. The molecular docking data show that the Coumarin and its derivative bind in subdomain IIA (site 1) of the HSA, nearby the Trp214 residue, that is in agreement with fluorescence quenching results. The binding mode obtained from the molecular docking demonstrates that thecarbon atoms C3 and C8 of the ligandsare oriented within the hydrophobic cavity of the site 1 of HSA, which also it was observed by the STD-NMR experiments. Overall, the experimental and theoretical data corroborate with each other and they are complementary.

\section{ACKNOWLEDGEMENTS.}

This work was supported by grant FAPESP proc :2009/53989-4

\section{References:}

[1] A. Drzewiecka, A.E. Koziol, M. Struga, T.PRuiz, M.F. Gomez, T. Lis, Structural characterization of derivatives of 4methylcoumarin - Theoretical and experimental studies, J Mol Structure. 1043 (2013) 109-115.

[2] N. Farinola, N. Piller, Pharmacogenomics: its role in reestablishing coumarin as treatment for lymphedema, Lymphat Res Biol. 3 (2005) 81-86. 
[3] R. Singh, B. Singh, S. Singh, N. Kumar, S. Kumar, S. Arora, Umbelliferone - An antioxidant isolated from Acacia nilotica (L.) Willd, Ex. Del. Food Chem. 120 (2010) 825-830.

[4] A. Witaicenis, L.N.Seito, L.C. Di Stasi, Intestinal antiinflammatory activity of esculetin and 4-methylesculetin in the trinitrobenzenesulphonic acid model of rat colitis, ChemicoBiological Interactions. 186 (2010) 211-218.

[5] A. Witaicenis, L.N. Seito, A. da SilveiraChagas, L.D. de Almeida Jr, A.C.Luchini, P. Rodrigues-Orsi, S.H. Cestari, L.C. Di Stasi, Antioxidant and intestinal anti-inflammatory effects of plantderivedcoumarin derivatives, Phytomedicine. 21 (2013) 240-246.

[6] A. Lacy, R. O'Kennedy, Studies on coumarins and coumarin related compounds to determine their therapeutic role in the treatment of cancer, Curr. Pharm. Des. 10 (2004) 3797-3811.

[7] D.P. Yeggoni, M. Gokara, D.M. Manidhar,A. Rachamallu, S. Nakka, C.S. Reddy, R. Subramanyam, Binding and Molecular Dynamics Studies of 7Hydroxycoumarin Derivatives with Human Serum Albumin and Its Pharmacological Importance, Mol. Pharmaceutics. 11 (2014) 1117-1131.

[8] C. Xiao, X.Y. Luo, D.J. Li, H. Lu, Z.Q. Liu, Z.G. Song, Y.H. Jin, Synthesis of 4-methylcoumarin derivatives containing 4,5dihydropyrazole moiety to scavenge radicals and to protect DNA, Eur J Med Chem. 53 (2012) 159-167.

[9] O. Dömötör, T. Tuccinardi, D. Karcz, M. Walsh, B.S. Creaven, E.A. Enyedy, Interaction of anticancer reduced Schiff base coumarin derivatives with human serum albumin investigated by fluorescence quenching and molecular modeling, Bioorg. Chem. 52 (2014) 16-23. [10] G.A. Ascoli, E. Domenici, C. Bertucci, Drug binding to human serum albumin: abridged review of results obtained with highperformance liquid chromatography and circular dichroism. Chirality. 18 (2006) 667-679.

[11] T. Peters Jr, All About Albumin: biochemistry, genetics and medical application, Academinc Press, San Diego, CA, 1995.

[12] S.M.T. Shaikh, J.Seetharamappa, P.B. Kandagal, D.H. Manjunatha, S.Ashoka, Spectroscopic investigations on the mechanism of interaction of bioactive dye with bovine serum albumin, Dyes and Pigments. 74 (2007) 665-671.

[13] S. Deepa, A.K. Mishra, Fluorescence spectroscopic study of serum albumin-bromadiolone interaction: fluorimetric determination of bromadiolone, J Pharm Biomed Anal. 38 (2005) 556-563.

[14] I.P. Caruso, W. Vilegas, M.A. Fossey, M.L. Cornélio, Exploring the binding mechanism of Guaijaverin to human serum albumin: Fluorescence spectroscopy and computational approach, Spectrochim. Acta Part A. 97 (2012) 449-455.

[15] R. Yadav, S. Das, P. Sen, Static and dynamic aspects of supramolecular interactions of Coumarin 153 and fluorescein with Bovine Serum Albumin, Aust. J. Chem. 65 (2012) 1305-1313.

[16] A. Maity, P.Mukherjee, T.Das, P.Ghosh, P.Purkayastha, Förster resonance energy transfer between pyrene and bovine serum albumin: effect of the hydrophobic pockets of cyclodextrins, Spectrochim. Acta Part A. 92 (2012) 382-387.

[17] A. Viegas, J. Manso, F.L. Nobrega, E.J. Cabrita, Saturation Transfer Difference (STD) NMR: A simple and fast method for ligand screening and characterization of protein binding, Journal of Chemical Education. 88 (2011) 990-994.

[18] N. Zaidi, E. Ahmad, M. Rehan, G. Rabbani, M.R. Ajmal, Y Zaidi, N. Subbarao, R.H. Khan, Biophysical insight into furosemide binding to human serum albumin: a study to unveil its impaired albumin binding in uremia, The Journal of Physical Chemistry. 117 (2013) 2595-2604.
[19] J. Shobini, A.K. Mishra, K. Sandhya, N. Chandra, Interaction of coumarins derivatives with human serum albumin: investigation by fluorescence spectroscopic technique and modeling studies, SpectrochimicaActa part A. 57 (2001) 1133-1147.

[20] R. Gonçalves, N. Mateus, I. Pianet, M. Laguerre, V. de Freitas, Mechanisms of tannin induced trypsin inhibition: A molecular approach, Langmuir. 27 (2011) 13122-13129.

[21] B. Meyer, T. Peters, NMR spectroscopy techniques for screening and identifying ligand binding to protein receptors,AngewChemInt Ed Engl. 42 (2003) 864-890.

[22] C. A. Lepre, J. M. Moore, J. W. Peng, Theory and Applications of NMR-Based Screening in Pharmaceutical Research, Chem. Rev. 104 (2004) 3641-3675.

[23] M. Mayer, B.J. Meyer, Characterization of Ligand Binding by Saturation Transfer Difference NMR Spectroscopy, Angew. Chem. Int. 38 (1999) 1784-1788.

[24] M. Mayer, B.J. Meyer, Group Epitope Mapping by Saturation Transfer Difference NMR To Identify Segments of a Ligand in Direct Contact with a Protein Receptor, Am. Chem. Soc. 25 (2001) 6108-6117.

[25] N.D. Chasteen, J.K. Grady, C.E. Holloway, Characterization of the binding, kinetics, and redox stability of vanadium (IV) and vanadium (V) protein complexes in serum, Inorg. Chem. 25 (1986) $2754-2760$

[26] C. Luchinat, G. Parigi, E. Ravera, NMR Technology: The competitive world of RAS biology, Nat Chem Biol. 10 (2014) 173 174

[27] I.E. Borissevitch, More about the inner filter effect: corresctions of Stern-Volmer fluorescence quenching constants are necessary at very low optical absorption of the quencher, J. Lumin. 81 (1999) 219-224.

[28] H.M. Berman, J. Westbrook, Z. Feng, G. Gilliland, T.N. Bhat, H. Weissig, I.N. Shindyalov, P.E. Bourne,"The Protein Data Bank." Nucleic Acids Research. 28 (2000) 235-242.

[29] S. Sugio, A. Kashima, S. Mochizuki, M. Noda, K. Kobayashi, Crystal structure of human serum albumin at 2,5 $\AA$ resolution, Protein Eng. 12 (1999) 439-446.

[30] M.F. Sanner, Python: a programming language for software integration and development. J. Mol. Graph Model. 17 (1999) 57 61.

[31] J. Angulo, P.M. Enríque-Navas, P.M. Nieto, Ligand-receptor binding affinities from saturation transfer difference (STD) NMR spectroscopy: the binding isotherm of STD initial growth rates, Chemistry. 26 (2010) 7803-12.

[32] J.R. Lakowicz, Principles of Fluorescence Spectroscopy, 2nd ed. Kluwer Academic Publishers/Plenum Press, New York, 1999.

[33] J.R. Lakowicz, G. Weber, Quenching of Fluorescence by Oxygen. A Probe for Structural Fluctuations in Macromolecules, Biochemistry 12 (1973) 4161-4170.

[34] Y.J. Hu, Y. Liu, J.B. Wang, X.H. Xiao, S.S. Qu, Study of the interaction between MonoammoniumGlycyrrhizinate and Bovine Serum Albumin, J. Pharm. Biom. Anal. 36 (2004) 915-919.

[35] B.P. Kamat, J. Seetharamappa, D. Kovala-Demertzi, Spectrofluorometric Study of the Interaction of Coumarin Derivatives with Bovine Serum Albumin, Journal of Photoscience. 11(2004), 65-69.

[36] W. R. Ware, Oxygen Quenching of Fluorescence in Solution: An Experimental Study of the Diffusion Process, J. Phys. Chem. 66 (1962) 455-458. 
Investigation of the Interaction between Coumarins and Its Derivatives with Human Serum Albumin: STD-NMR, Fluorescence and Docking Molecular Studies

a)

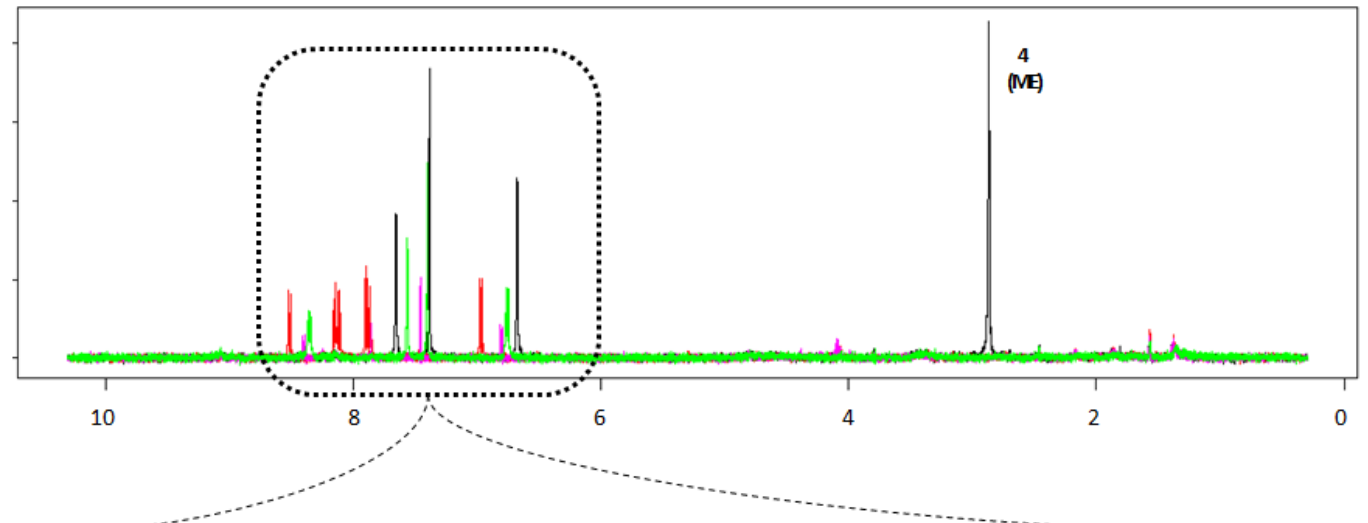

b)

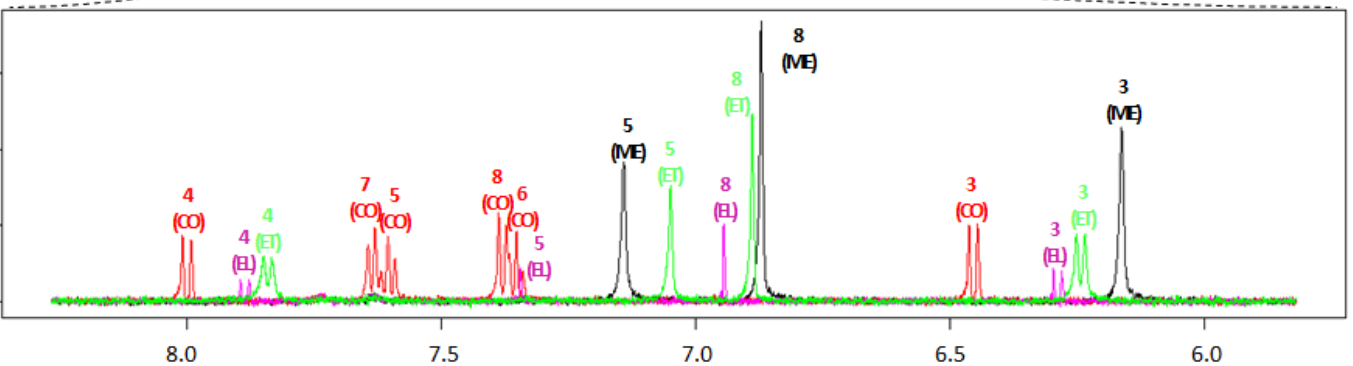

c)

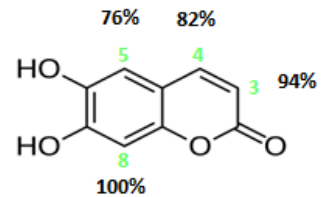

Esculetin

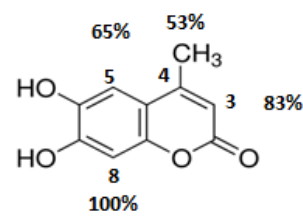

4-Methylesculetin

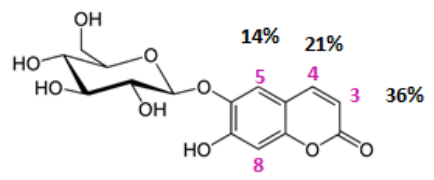

$100 \%$

Esculin<smiles>CC(C)(C)[13CH3]</smiles>

Coumarin
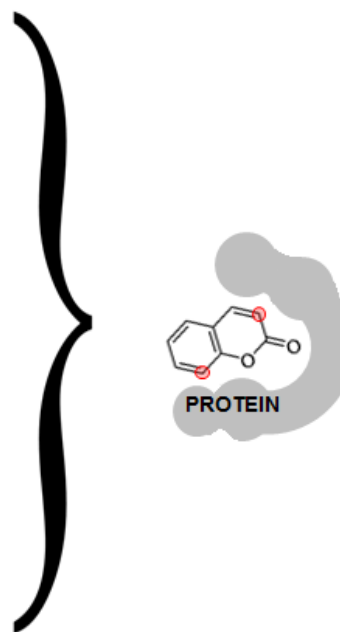

Figure 1.Superpose of saturation transfer difference spectra of HSA with 4-Methylesculetin (MEblack), Esculetin (ET-green), Coumarin (CO-red) and Esculin (EL-pink) (a). Zoom in the low field region, showing signals of each ligand (b). Chemical structures of coumarins and its derivatives following the color code in the spectra and relative STD amplification factors as percentages for group mapping epitope (c). A binding mode is proposed by using the binding epitopes data. 
Investigation of the Interaction between Coumarins and Its Derivatives with Human Serum Albumin: STD-NMR, Fluorescence and Docking Molecular Studies
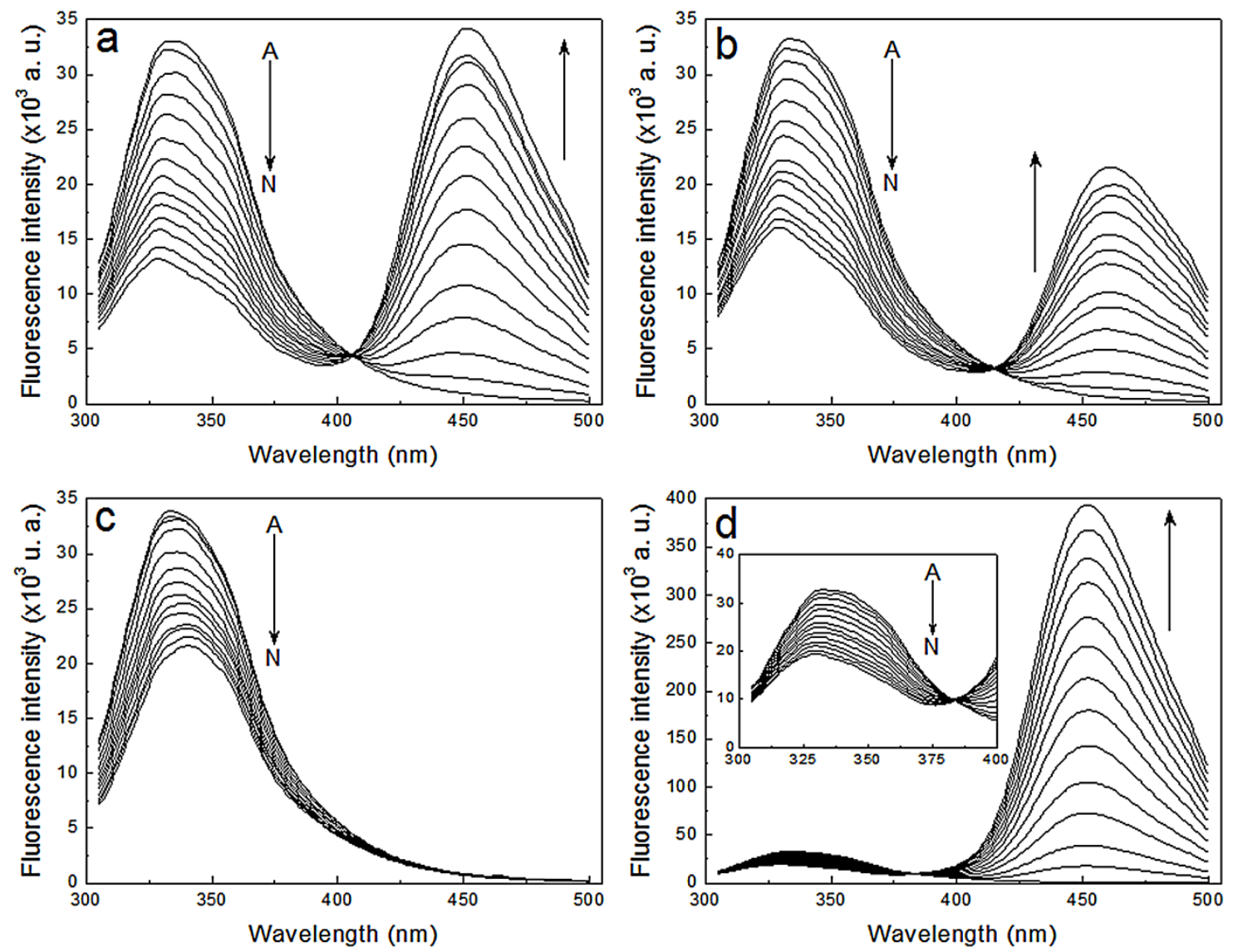

Figure 2. Emission spectra of the HSA with the increment of the (a) 4-Methylesculetin, (b) Esculetin, (c) Coumarin and (d) Esculin concentration $\left(\mathrm{T}=298 \mathrm{~K}, \lambda_{\mathrm{ex}}=295 \mathrm{~nm}\right)$. [HSA] $=10 \mu \mathrm{M}$; [Ligand] $(\mu \mathrm{M}), \mathrm{A}-\mathrm{N}$ : from 0 to 25 with the increments of 2.5. The insert in (d) corresponds to the spectral range (300-400 nm) of emission of HSA.

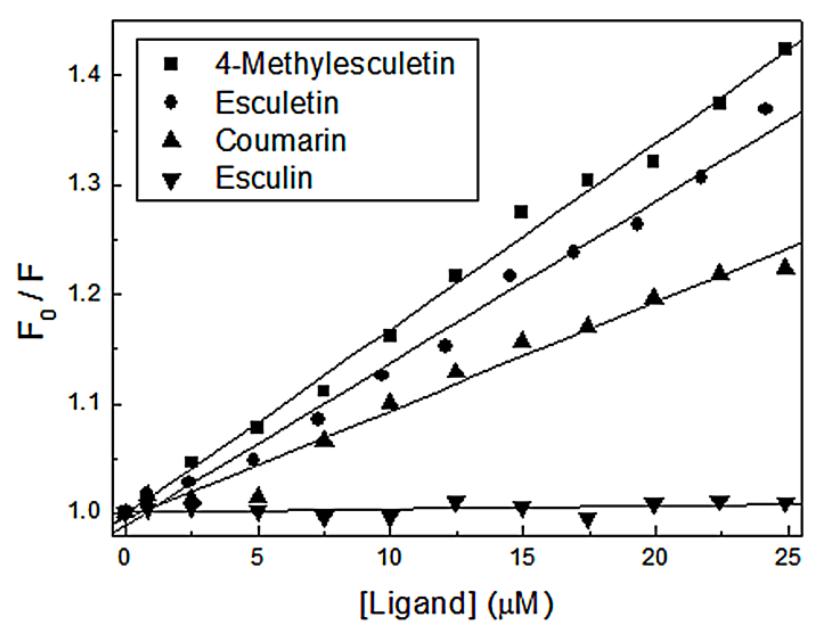

Figure 3. Stern-Volmer plots for the fluorescence quenching of the HSA $(10.0 \mu \mathrm{M})$ by ligands at $\mathrm{pH} 7.0$ and $298 \mathrm{~K}$. 
Investigation of the Interaction between Coumarins and Its Derivatives with Human Serum Albumin: STD-NMR, Fluorescence and Docking Molecular Studies
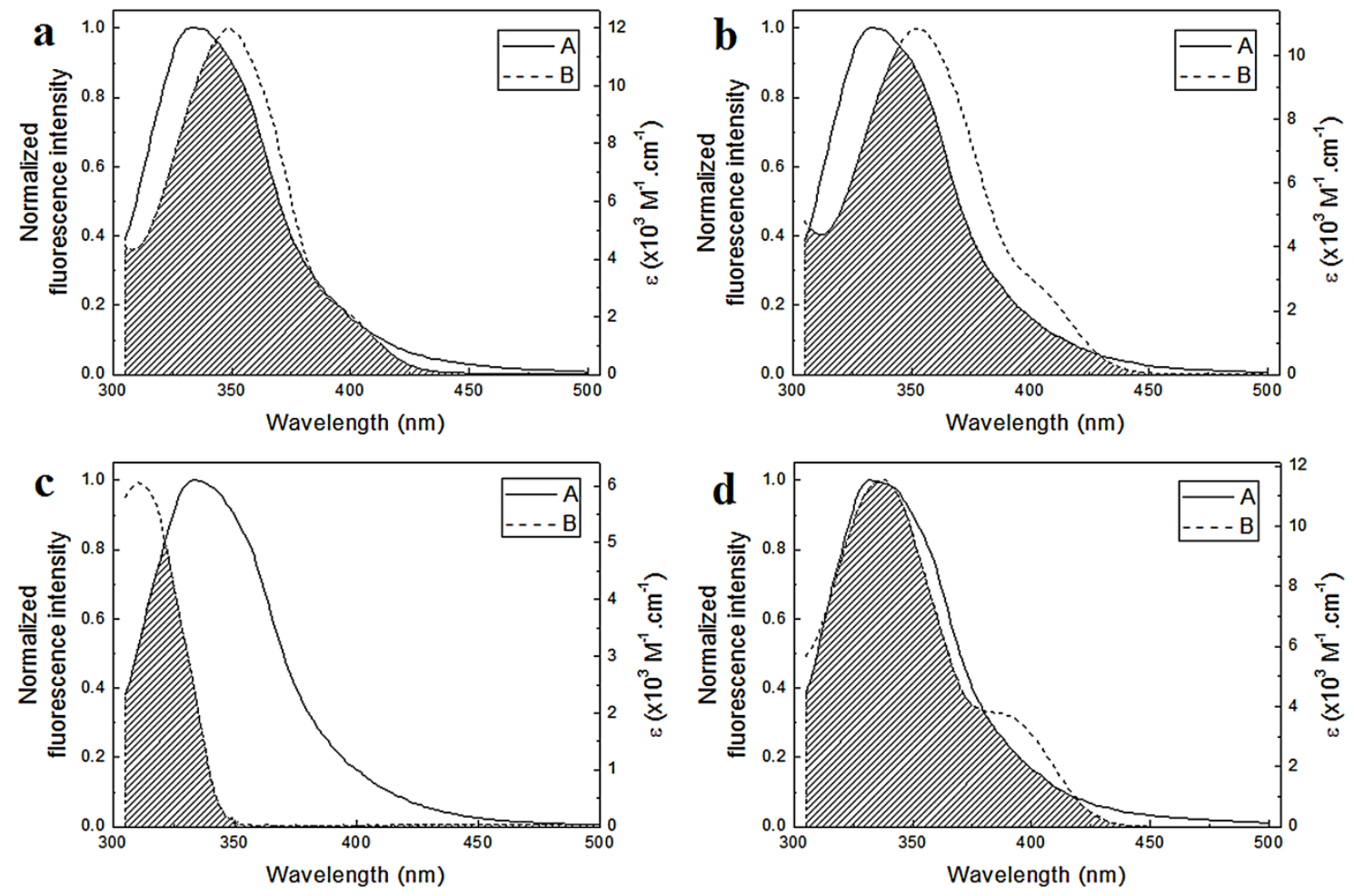

Figure 4. Spectral overlaps between the HSA fluorescence (A) and the ligands absorption (B) with 4Methylesculetin (a), Esculetin (b), Coumarin (c) and Esculin (d).

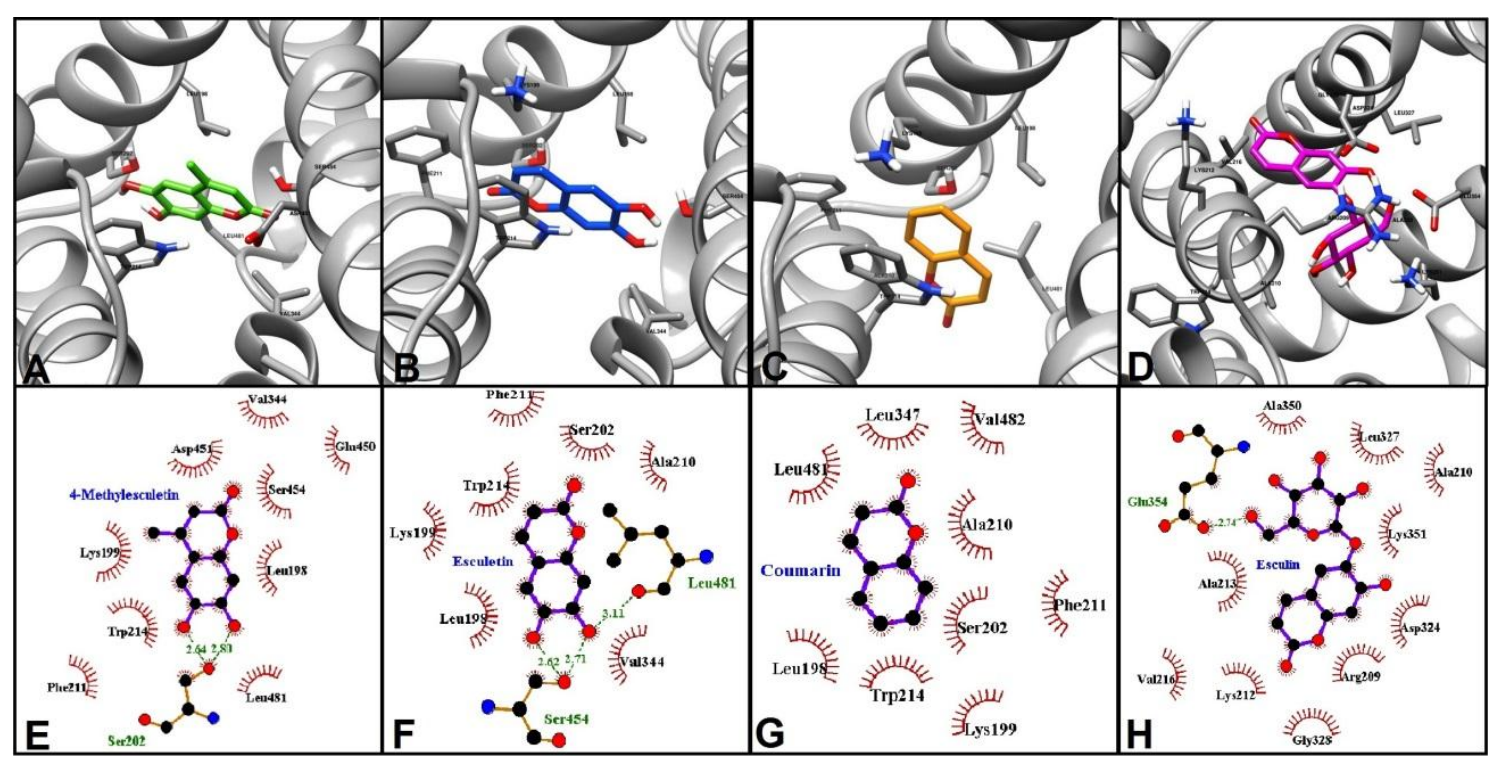

Figure 5. Docking pose of 4-Methylesculetin (green), Esculetin (blue), Coumarin (orange) and Esculin (magenta) in HSA. 4-Methylesculetin, Esculetin and Coumarin docked in an inner pocket near Trp214 (A, B and C), while Esculin docked on the protein surface (D). 4-Methylesculetin (E), Esculetin (F) and Esculin (H) makes hydrogen bonds and hydrophobic interaction with the protein, while Coumarin $(\mathrm{G})$ makes only hydrophobic interaction. 
Investigation of the Interaction between Coumarins and Its Derivatives with Human Serum Albumin: STD-NMR, Fluorescence and Docking Molecular Studies

Table S1: Cluster of the ligands docked into HSA. It was generated 100 poses per ligand. Each cluster had a low binding energy variation between their poses.

\begin{tabular}{|c|c|c|c|}
\hline Ligand & Number of groups & Poses per group & $\begin{array}{c}\text { Binding energy } \\
\text { variation (kcal/mol) }\end{array}$ \\
\hline \multirow{3}{*}{ 4-Methylesculetin } & \multirow{3}{*}{3} & 81 & 0.009 \\
\hline & & 18 & 0.12 \\
\hline & & 1 & - \\
\hline \multirow{4}{*}{ Esculetin } & \multirow{4}{*}{4} & 89 & 0.05 \\
\hline & & 6 & 0.13 \\
\hline & & 4 & 0.01 \\
\hline & & 1 & - \\
\hline Coumarin & 1 & 100 & 0.001 \\
\hline \multirow{12}{*}{ Esculin } & \multirow{12}{*}{12} & 58 & 1.21 \\
\hline & & 9 & 1.64 \\
\hline & & 7 & 0.31 \\
\hline & & 5 & 0.22 \\
\hline & & 4 & 0.60 \\
\hline & & 4 & 0.95 \\
\hline & & 4 & 1.18 \\
\hline & & 3 & 0.99 \\
\hline & & 3 & 0.35 \\
\hline & & 1 & - \\
\hline & & 1 & - \\
\hline & & 1 & - \\
\hline
\end{tabular}

Table S2: Binding energy of each molecule from each group docked in HSA. The binding energy and specific interactions are given in $\mathrm{kcal} / \mathrm{mol}$.

\begin{tabular}{cccccc}
\hline Molecule & Cluster & $\begin{array}{c}\text { Binding } \\
\text { energy }\end{array}$ & $\begin{array}{c}\text { Hydrophobic } \\
\text { energy }\end{array}$ & $\begin{array}{c}\text { Hydrogen } \\
\text { bond }\end{array}$ & $\begin{array}{c}\text { Electrostatic } \\
\text { energy }\end{array}$ \\
\hline $4-$ & 1 & -6.01 & -2.168 & -4.132 & -0.31 \\
Methylesculetin & 2 & -5.94 & -5.681 & -0.659 & -0.2 \\
\hline \multirow{2}{*}{ Esculetin } & 1 & -5.65 & -2.692 & -3.358 & -0.19 \\
& 2 & -5.48 & -2.508 & -3.512 & -0.06 \\
\hline Coumarin & 3 & -5.3 & -5.768 & -0.052 & -0.08 \\
\hline Esculin & 4 & -5.09 & -1.76 & -3.44 & -0.49 \\
\hline & 1 & -5.79 & -5.81 & 0 & 0.02 \\
\hline & 2 & -5.71 & -0.455 & -5.065 & -0.19 \\
& & -5.87 & -3.181 & -1.939 & -0.75 \\
\hline
\end{tabular}




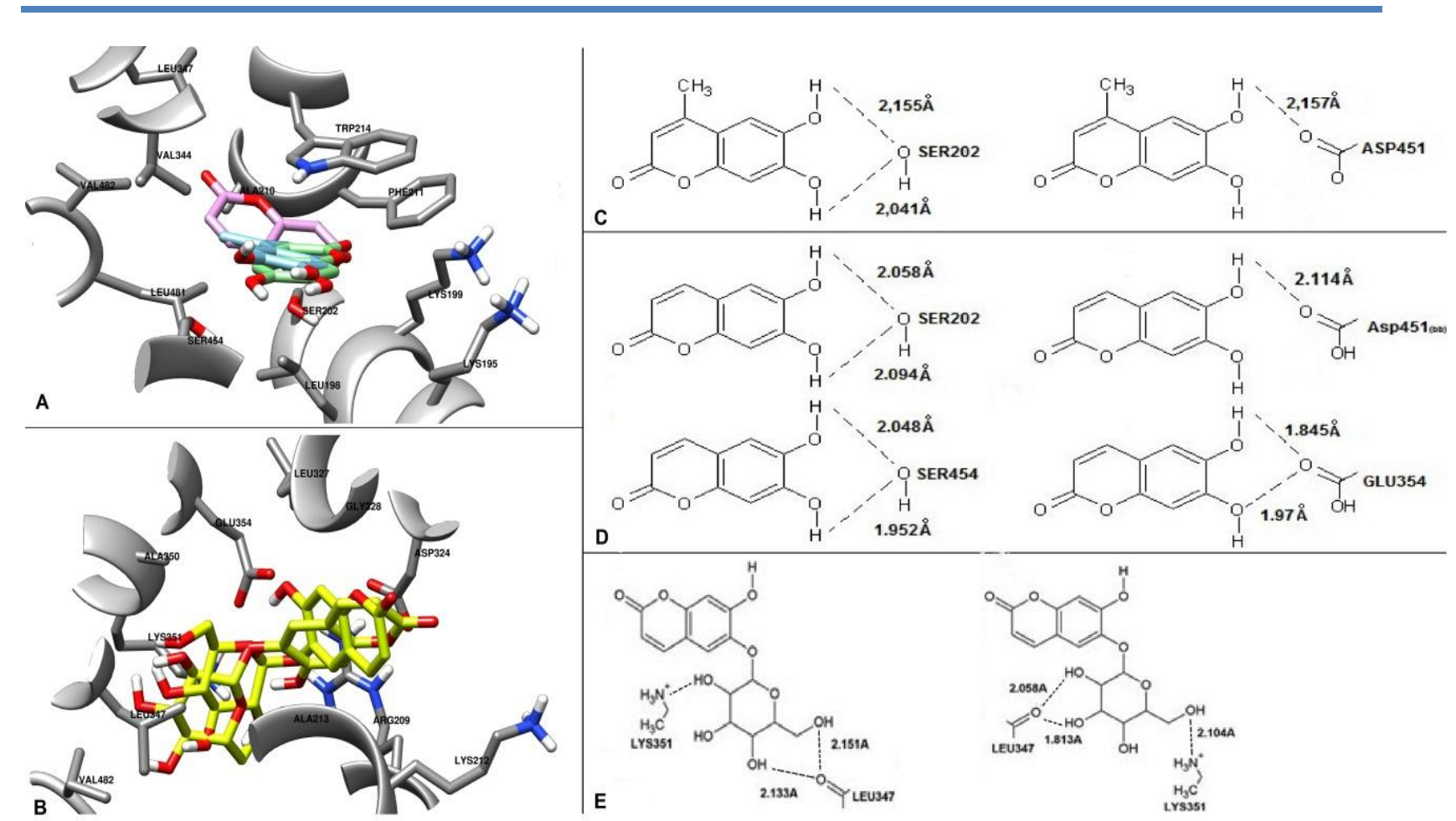

Figure S1: Docking pose of 4-Methylesculetin (light blue), Esculetin (green), Coumarin (magenta) and Esculin (yellow) in HSA. 4-Methylesculetin, Esculetin and Coumarin docked in an inner pocket near Trp214 (A), while Esculin docked on the protein surface (B). 4-Methylesculetin (C), Esculetin (D) and Esculin (E) makes hydrogen bonds with the protein. 